\title{
COMPARISON OF WEDGED HEPATIC VEIN PRESSURE WITH \\ PORTAL VEIN PRESSURE IN HUMAN SUBJECTS WITH \\ CIRRHOSIS ${ }^{1,2}$
}

\author{
By TELFER B. REYNOLDS, DONALD C. BALFOUR, JR., DAVID C. LEVINSON, \\ WILLIAM P. MIKKELSEN, AND ARTHUR C. PATTISON
}

(From the Departments of Medicine and Surgery, University of Southern California School
of Medicine, and the Los Angeles County Hospital, Los Angeles, Calif.)

(Submitted for publication July 27, 1954; accepted October 27, 1954)

A method of assessing portal venous pressure in animals by wedging a cardiac catheter into a peripheral hepatic venule was described by Myers and Taylor (1) and by Friedman and Weiner (2). The application of this technique in humans has been reported by Myers and Taylor (1), Paton, Reynolds, and Sherlock (3), and Krook (4). It seems clear from the evidence presented that the presence of portal hypertension can be reliably predicted from the finding of an elevated wedged hepatic vein pressure (WHVP). Paton, Reynolds, and Sherlock have shown that in patients without liver disease WHVP measures only a few $\mathrm{mm}$. $\mathrm{Hg}$ more than right atrial pressure (3). Likewise in non-cirrhotic liver disorders not associated with portal hypertension WHVP is normal (3). By contrast in a large number of cirrhotic subjects reported by the aforementioned three groups of investigators WHVP was invariably elevated to some degree.

This technique would be more valuable if it were known how closely the pressure measured in the wedged position corresponded to actual portal venous pressure (PVP). In cats (1) and in dogs (2) WHVP was found to be almost identical with PVP both in the normal animal and when both pressures had been elevated by epinephrine injection. In two human subjects with portal hypertension, PVP, measured postoperatively through a polyethylene tube leading from an omental vein, compared favorably with WHVP measured preoperatively (3). To our knowl-

\footnotetext{
1 Preliminary results reported before the Western Surgical Association, December 3, 1953, and the Western Section of the American Federation for Clinical Research, January 28, 1954.

2 Aided by grants from the National Heart Institute, U. S. Public Health Service (H-1718) and the Los Angeles County Heart Association.
}

edge no other studies have been reported comparing WHVP and PVP in man.

This communication describes a number of such comparisons the preliminary results of which have been published in abstract form (5).

\section{METHODS AND MATERIAL}

We have followed the technique originally described by Warren and Brannon (6) and elaborated by Bradley, Ingelfinger, Bradley, and Curry (7) for catheterization of the hepatic vein. A cardiac catheter is passed from the median antecubital vein of either arm under fluoroscopic guidance into the right atrium. We have had the best results with moderately stiff catheters, No. 8 or No. 9 gauge, prepared straight except for the usual curved tip. Right atrial pressure is recorded with a Statham strain gauge located $5 \mathrm{~cm}$. posterior to the sternal angle with the patient supine. The catheter is then turned so that the curved tip is pointed to the right and posteriorly in order to enter the inferior vena cava. This may require considerable manipulation in subjects with cirrhosis because of the unusual location of the caval orifice incident to hepatic enlargement. The catheter tip will usually enter a right sided hepatic vein if it is advanced 1 to $2 \mathrm{~cm}$. beyond the cavo-auricular junction; if it does not do so the tip can be rotated anteriorly and the wall of the vena cava explored again. The catheter seldom enters the hepatic vein draining the left lobe. The right renal vein can be mistaken for an hepatic vein, however, it forms almost a right angle with the vena cava, it opens more caudad than most hepatic veins, in the lateral view it courses more posteriorly, and the blood withdrawn from it contains more oxygen. After entering an hepatic vein the catheter is advanced as far as possible into the liver substance until it is wedged in a small hepatic venule. When properly wedged it will usually be far out in the periphery of the liver (an anterior course can be checked fluoroscopically). When it is withdrawn slight tension will be required to free the tip from the wedged position from which it will spring suddenly. Unfortunately there is no way of being absolutely certain that the catheter is properly wedged; if it becomes lodged at a venous bifurcation and blood flow is not completely obstructed an accurate wedge pressure will not be recorded. For this reason it is advisable to make pressure records from 
more than one site in the liver if possible. After the wedged position is attained pressure is measured and a continuous recording made as the catheter is withdrawn to the free hepatic vein. Inferior vena caval pressure and the gradient between inferior cava and the right atrium are recorded at the end of the procedure.

Since changes in the general level of venous pressure would be expected to produce equivalent changes in wedged hepatic vein and portal vein pressures regardless of the presence or absence of hepatic disease, WHVP and PVP have been expressed as net pressure above right atrial level. This has the effect of eliminating elevations in wedged pressure (and presumably portal pressure) such as have been shown to accompany right atrial pressure rises in heart failure (3). In this study when actual right atrial pressure could be recorded it was subtracted from measured wedged pressure and portal pressure to obtain WHVP and PVP. When actual right atrial pressure could not be measured (as in the patients at surgery) it was assumed to be zero and the strain gauge was located externally at the estimated right atrial level.

PVP was recorded by inserting a 21 gauge needle into the exposed portal vein perpendicularly to the long axis of the vein. The needle was connected to the strain gauge by a polyethylene tube of inside diameter $1.2 \mathrm{~mm}$.

The 19 patients on whom the pressure comparisons were made had varying degrees of portal hypertension due to alcoholic cirrhosis. None had clinically evident ascites. Seventeen of them were operated on for the performance of a portacaval shunt though it was not performed in all instances. Two were operated on for other intra-abdominal procedures. The pressure comparisons were made in three different ways.

Group $A$. Two patients were operated upon for conditions other than their portal hypertension. At the time of surgery polyethylene tubing was secured in a mesenteric vein, brought to the outside through a stab wound in the anterior abdominal wall and kept patent by a slow infusion. This procedure has previously been performed without harm to the patient $(3,8)$. Three days postoperatively the hepatic vein was catheterized and WHVP was recorded, followed immediately by a pressure recording from the mesenteric vein via the polyethylene tubing. The strain gauge was maintained in the same position $(5 \mathrm{~cm}$. below the sternal angle with the patient supine) for all measurements. Both mesenteric vein pressure and wedged pressure were corrected by subtracting right atrial pressure.

Group B. Seven patients who underwent portacaval anastomosis were catheterized immediately preoperatively. WHVP was measured and corrected by subtracting right atrial pressure. From one to three hours later, in the operating room, the pressure in the portal vein itself was recorded. The estimated right atrial position $(5 \mathrm{~cm}$. posterior to the sternal angle) was used as a zero reference point for recording PVP.

Group $C$. Ten patients were catheterized immediately preceding operation for portacaval anastomosis. The catheter was left in the wedged hepatic vein and kept patent with a continuous slow infusion while the patient was taken to surgery. When the portal vein was exposed WHVP was recorded, followed immediately by PVP. Both recordings were made with the strain gauge at the same zero reference point-the externally estimated position of the right atrium. In five of the patients WHVP was also measured at the time of catheterization, prior to the administration of anaesthesia.

\section{Complications}

Catheterization of the hepatic vein appears to be an innocuous procedure. The danger points of cardiac catheterization (the coronary sinus, tricuspid valve, right ventricle) are avoided. Arrhythmias are minor or absent. There have been no untoward results in the authors' experience except for an occasional mild thrombophlebitis or infection at the cut-down site. The hepat:c vein is easily entered in the normal subject. However, in patients with cirrhosis of the liver some difficulty may be encountered, necessitating an experienced operator and considerable manipulation of the catheter. We require an average of two minutes of fluoroscopic time per patient and have failed to enter the hepatic vein in 5 of over 70 attempts in cirrhotic patients.

\section{RESULTS}

In every instance WHVP was elevated and as the catheter was withdrawn to the free hepatic vein the abrupt drop in pressure characteristic of intrahepatic portal hypertension was seen (Figure 1). This abrupt pressure drop was never seen in patients who did not have hepatic cirrhosis.

Table I and Figure 2 depict the results of the pressure comparisons in the 19 patients. In every case WHVP was lower than PVP. However, in no instance was there a major discrepancy between the two readings.

In the two patients in Group A WHVP was 4 and 8 per cent below mesenteric vein pressure.

In the seven cases in Group B WHVP aver-

\section{PORTAL CIRRHOSIS}

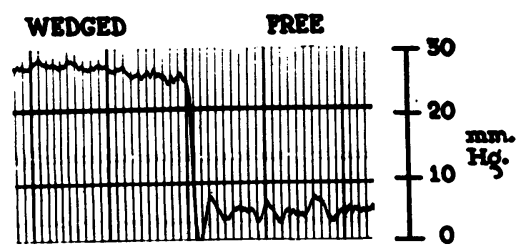

Fig. 1. Abrupt Drop in Pressure as the Catheter is Withdrawn from a "Wedged" Position in the Hepatic Vein to a "Free" Position 
TABLE I

Wedged hepatic, portal and mesenteric vein pressures in 19 subjects with cirrhosis

\begin{tabular}{|c|c|c|c|c|c|}
\hline GROUP & $\begin{array}{c}\text { CASE } \\
\text { NO. }\end{array}$ & $\begin{array}{l}\text { W.H.V.P. } \\
\text { PRE-OPERATIVE } \\
\text { (Fi.He obove } \\
\text { rt.otrium) }\end{array}$ & 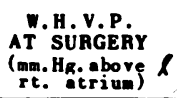 & $\begin{array}{l}\text { P.V.P. } \\
\underset{\text { AT SURGERY }}{\text { (mm. Hg.ebore } l} \\
\text { rt.atrium) }\end{array}$ & $\begin{array}{l}\text { MESENTERIC } \\
\text { V.P. } \\
\text { (ma. HR.eboye } \\
\text { st.otriug) }\end{array}$ \\
\hline \multirow[t]{2}{*}{$\mathbf{A}$} & 1 & $19.0 *$ & & & 21.0 \\
\hline & 2 & $20.0 *$ & & & 21.0 \\
\hline \multirow[t]{8}{*}{$\bar{B}$} & 3 & 12.0 & & 14.0 & \\
\hline & 4 & 17.0 & & 20.0 & \\
\hline & 5 & 20.0 & & 21.0 & \\
\hline & 6 & 21.0 & & 24.0 & \\
\hline & 7 & 26.0 & & 29.0 & \\
\hline & 8 & 28.0 & & 31.0 & \\
\hline & 9 & 28.0 & & 32.0 & \\
\hline & Meene & 21.7 & & 24.4 & \\
\hline \multirow[t]{11}{*}{ C } & 10 & & 13.0 & 14.0 & \\
\hline & 11 & & 13.0 & 15.0 & \\
\hline & 12 & 15.5 & 13.5 & 17.0 & \\
\hline & 13 & 19.5 & 15.0 & 20.0 & \\
\hline & 14 & 20.5 & 17.0 & 22.0 & \\
\hline & 15 & 24.0 & 21.0 & 26.0 & \\
\hline & 16 & & 21.0 & 29.0 & \\
\hline & 17 & & 22.0 & 29.0 & \\
\hline & 18 & & 23.0 & 25.0 & \\
\hline & 19 & 27.0 & 24.0 & 30.0 & \\
\hline & Means & & 18.3 & 22.7 & \\
\hline
\end{tabular}

- performed simultencously with mesenteric vein pressure measurements - see text.

*ctual rt. etrial pressure measured and subtracted.

$l$ strain gauge located at externally estimated rt. atrial level.

aged 11 per cent (range 5 to 15 per cent) below PVP.

In the 10 patients in Group C WHVP averaged 20 per cent (range 9 to 28 per cent) below PVP. A typical example is pictured in Figure 3 . The greatest discrepancy observed was in a patient in whom WHVP measured $21 \mathrm{~mm}$. $\mathrm{Hg}$ and PVP measured $29 \mathrm{~mm}$. $\mathrm{Hg}$.

In the combined group of 19 patients WHVP averaged 15.3 per cent below PVP.
B.

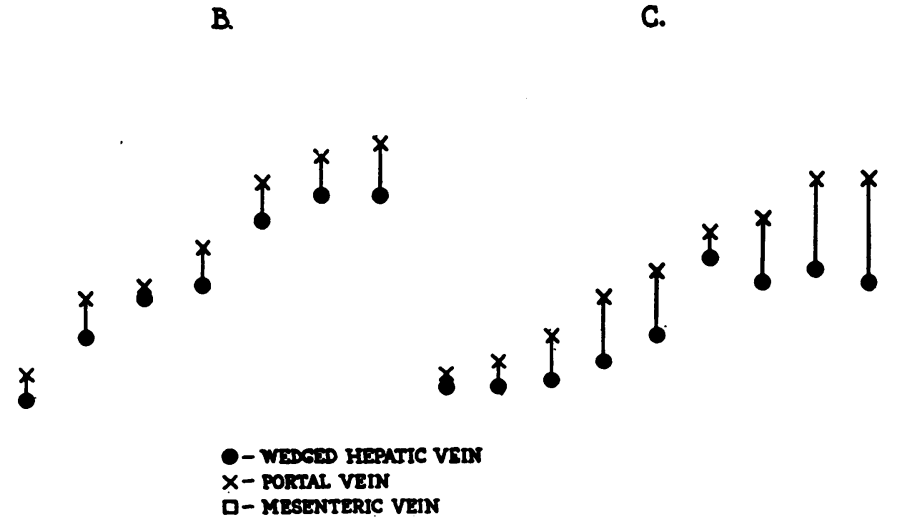

C. $x$ - PORThL Vens
A.

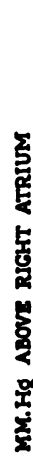

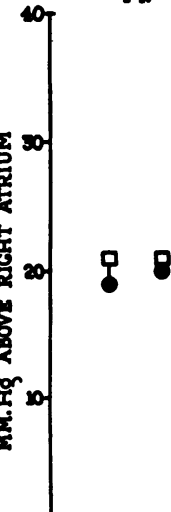

Fig. 2. Comparisons of Wedged Hepatic Vein and Portal or Mesenteric Vein Pressures in Three Groups of Patients with Cirrhósis

For description of the groups see text. 


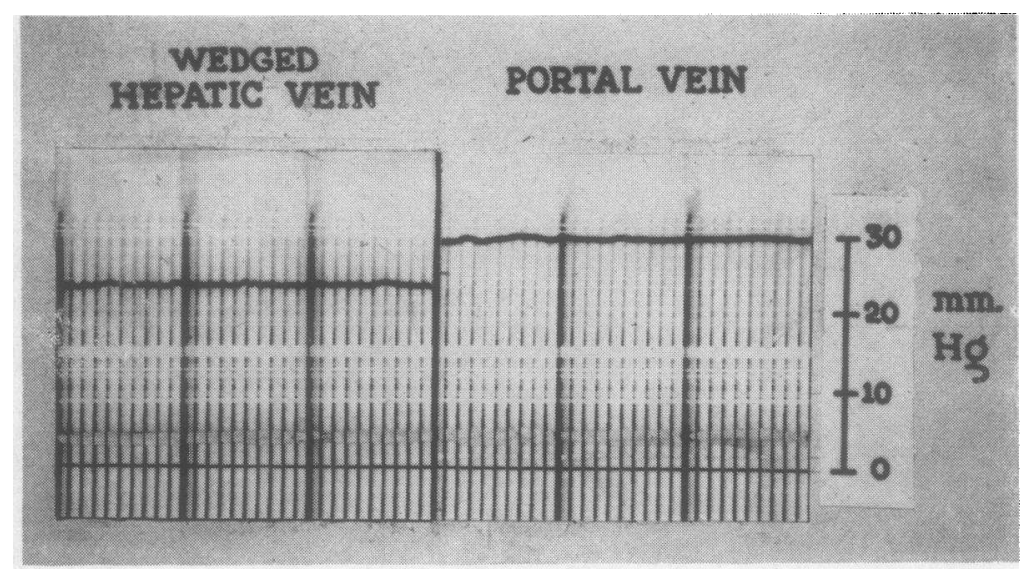

Fig. 3. Comparison of Wedged Hepatic Vein and Portal. Vein Pressures Made at Surgery

\section{DISCUSSION}

There are some theoretical objections to the pressure comparisons in Group $\mathrm{A}$ and $\mathrm{B}$. In the two patients in Group $A$, the polyethylene was fixed in small mesenteric veins. It is possible that some degree of blood clotting occurred in this relatively static system in spite of the continuous infusion. A small clot near the end of the tubing might have interfered with accurate pressure recording.

In Group B the two pressure recordings were made under different circumstances. At the time of the PVP recording the patients were anaesthetized. Anaesthesia has been shown to lead to a fall in splanchnic blood flow in patients without liver disease $(9,10)$. This could be due either to splanchnic or intrahepatic vasoconstriction or both. If the former exceeded the latter, a fall in PVP would be expected. Such a situation might obtain in cirrhosis with a relatively fixed intrahepatic vasculature. PVP would then drop, accounting for the closer agreement between WHVP and PVP in our patients in Group $B$ than in Group C. In fact, in each of the five patients in Group C in whom WHVP was measured both preoperatively and at surgery the second determination was lower, the mean drop being 15 per cent (Table I). This would be compatible with a fall in splanchnic blood flow secondary to splanchnic vasoconstriction.

Exact comparison between WHVP and PVP in the patients in Group $B$ is also rendered unreliable by the possibility of small errors in position- ing of the external zero reference point used for measuring PVP.

There seem to be no major objections to the validity of the comparisons in the patients in (iroup C. The same zero reference point was used for both pressures which were recorded by the same mechanism only seconds apart. It is true that a lateral pressure was recorded in the portal vein which neglects the kinetic energy of blood flow. The magnitude of this kinetic energy. though not measured, is assumed to be small because of the relatively low velocity of blood flow in the portal vein in cirrhosis.

Though there was considerable variability in the extent to which WHVP approached PVP, this variability was within a relatively narrow range and no instance of a marked discrepancy between the two pressures was encountered.

It is to be expected that pressure measured in the obstructed hepatic vein would be somewhat lower than portal pressure. The recording of portal pressure from the wedged catheter depends upon the presence of a static blood column between the portal inflow system and the catheter tip. Over this static column pressure can be transmitted without the usual decrement from vascular resistance. Although the wedged catheter does obstruct blood flow in a small segment of liver this obstruction is probably never complete because of collateral channels at or near the sinusoidal level. Hence WHVP should be consistently less the PVP. Hepatic arterial inflow into this relatively static vascular column could 
raise catheter tip pressure to arterial levels were it not for the possibility of free backflow into the portal system when the latter's pressure is exceeded.

The fact that WHVP closely approaches PVP indicates that the majority of the abnormal resistance to blood flow in the cirrhotic liver lies between the wedged catheter tip and the nearest freely anastomosing vascular channels distal to it. If there are freely anastomosing channels in the sinusoidal area, it would follow that the major resistance is post-sinusoidal in the small hepatic venules. If, however, there is a diffuse reduction in anastomosing vessels throughout the liver lobule then no such inference can be drawn.

Even with the catheter free in the hepatic vein, there may be some obstruction to blood flow through that radicle. This may result in the recording of a higher pressure from the free hepatic vein than from the inferior vena cava. For this reason we have chosen not to express WHVP as the difference between the pressure levels in the wedged and free positions. The use of an external zero reference point for recording WHVP favors inconsistency between observers because of the difficulty in locating the point exactly at atrial level. Furthermore it provides no means of distinguishing between elevations in WHVP secondary to a generalized rise in venous pressure (i.e., right heart failure) and elevations due to liver disease. The use of measured right atrial pressure as a zero reference point solves these problems. However, abnormal pressure gradients between inferior vena cava and right atrium will then be reflected in WHVP. We have found significant cavo-auricular pressure gradients in several patients with ascites (none in this series). In measuring PVP or WHVP we are chiefly interested in the resistance to blood flow through the liver, i.e., the pressure gradient between portal system and inferior vena cava. Although it is true that resistance to blood flow in the inferior vena cava itself carries no implication as far as liver disease is concerned, it is equally true that any increment in portal pressure provided by this resistance is important in the development of esophageal varices. Since varices are connections between the portal and superior vena caval systems any factors contributing to a pressure differential between the two should be important.
We have utilized right atrial pressure as a baseline for this reason. However, we believe inferior vena caval pressure should also be recorded since wedged pressure minus caval pressure gives the best picture of hepatic vascular resistance. It seems hardly necessary to emphasize that whether PVP or WHVP is used as an index of hepatic resistance the measurement is more valuable if it is accompanied by an estimate of liver blood flow.

A deterrent to the utilization of WHVP in a quantitative fashion is the lack of a definitive means of establishing that the catheter tip is properly wedged. We have occasionally, by continued manipulation, been able to advance the catheter tip further into the liver beyond the point at which it was originally thought to be wedged with the result that a higher WHVP was obtained. For this reason we emphasize the value of recording WHVP from more than one site.

\section{CONCLUSION}

From the data presented it appears likely that, in cirrhosis, WHVP represents a large and roughly predictable fraction of PVP. Further comparisons of these two measurements should establish whether this is true in all patients with cirrhosis.

Knowledge of the approximate portal pressure as determined by this technique has proven to be of clinical value in the diagnosis of obscure cases of cirrhosis (11), in assessing the need for portacaval anastomosis, in evaluating the merits of other procedures designed to ameliorate portal hypertension and in the diagnosis of extrahepatic portal hypertension.

\section{SUMMARY}

1. Portal vein pressure (PVP) and the pressure measured after wedging a catheter into a peripheral hepatic vein (WHVP) have been compared in 19 cirrhotic patients undergoing abdominal surgery.

2. WHVP correlated reasonably well with PVP in all instances. In 10 simultaneous comparisons WHVP averaged 20 per cent (range 9 to 28 per cent) below PVP.

3. In five patients WHVP averaged 15 per cent (range 13 to 23 per cent) lower at surgery than preoperatively. 


\section{ACKNOWLEDGMENT}

Facilities for catheterization were kindly made available to the investigators by George C. Griffith, M.D.

\section{REFERENCES}

1. Myers, J. D., and Taylor, W. J., An estimation of portal venous pressure by occlusive catheterization of an hepatic venule. J. Clin. Invest., 1951, 30, 662.

2. Friedman, E. W., and Weiner, R. S., Estimation of hepatic sinusoid pressure by means of venous catheters and estimation of portal pressure by hepatic vein catheterization. Am. J. Physiol., 1951, 165, 527.

3. Paton, A., Reynolds, T. B., and Sherlock, S., Assessment of portal venous hypertension by catheterization of hepatic vein. Lancet, 1953, 1, 918.

4. Krook, H., Estimation of portal venous pressure by occlusive hepatic vein catheterization. Scandinav. J. Clin. \& Lab. Invest., 1953, 5, 285.

5. Reynolds, T. B., Balfour, D. C., Jr., Levinson, D. C., Magidson, O., Mikkelsen, W. P., and Pattison, A. C., Measurement of portal venous pressure by hepatic vein catheterization. Clin. Res. Proc., 1954, 2, 148.

6. Warren, J. V., and Brannon, E. S., A method of obtaining blood samples directly from the hepatic vein in man. Proc. Soc. Exper. Biol. \& Med., 1948, 55, 144.

7. Bradley, S. E., Ingelfinger, F. J., Bradley, G. P., and Curry, J. J., The estimation of hepatic blood flow in man. J. Clin. Invest., 1945, 24, 890.

8. Bradley, S. E., Smythe, C. M., Fitzpatrick, H. F., and Blakemore, A. H., The effect of portacaval shunt on estimated hepatic blood flow and oxygen uptake in cirrhosis. J. Clin. Invest., 1953, 32, 526.

9. Habif, D. V., Papper, E. M., Fitzpatrick, H. F., Lowrance, P., Smythe, C. McC., and Bradley, S. E., The renal and hepatic blood flow, glomerular filtration rate, and urinary output of electrolytes during cyclopropane, ether, and thiopental anaesthesia, operation, and the immediate postoperative period. Surgery, 1951, 30, 241.

10. Shackman, R., Graber, I. G., and Melrose, D. G., Liver blood flow and general anaesthesia. Clin. Sc. 1953, 12, 307.

11. Reynolds, T. B., and Balfour, D. C., Jr., Hepatic vein catheterization in the diagnosis of cirrhosis. To be published. 